\title{
A Model-Theoretic Framework for Theories of Syntax
}

\author{
James Rogers \\ Institute for Research in Cognitive Science \\ University of Pennsylvania \\ Suite 400C, 3401 Walnut Street \\ Philadelphia, PA 19104 \\ jrogers@linc.cis . upenn.edu
}

\begin{abstract}
A natural next step in the evolution of constraint-based grammar formalisms from rewriting formalisms is to abstract fully away from the details of the grammar mechanism-to express syntactic theories purely in terms of the properties of the class of structures they license. By focusing on the structural properties of languages rather than on mechanisms for generating or checking structures that exhibit those properties, this model-theoretic approach can offer simpler and significantly clearer expression of theories and can potentially provide a uniform formalization, allowing disparate theories to be compared on the basis of those properties. We discuss $L_{K, P}^{2}$, a monadic second-order logical framework for such an approach to syntax that has the distinctive virtue of being superficially expressive - supporting direct statement of most linguistically significant syntactic properties - but having well-defined strong generative capacitylanguages are definable in $L_{K, P}^{2}$ iff they are strongly context-free. We draw examples from the realms of GPSG and GB.
\end{abstract}

\section{Introduction}

Generative grammar and formal language theory share a common origin in a procedural notion of grammars: the grammar formalism provides a general mechanism for recognizing or generating languages while the grammar itself specializes that mechanism for a specific language. At least initially there was hope that this relationship would be informative for linguistics, that by characterizing the natural languages in terms of languagetheoretic complexity one would gain insight into the structural regularities of those languages. Moreover, the fact that language-theoretic complexity classes have dual automata-theoretic characterizations offered the prospect that such results might provide abstract models of the human language faculty, thereby not just identifying these regularities, but actually accounting for them.

Over time, the two disciplines have gradually become estranged, principally due to a realization that the structural properties of languages that characterize natural languages may well not be those that can be distinguished by existing language-theoretic complexity classes. Thus the insights offered by formal language theory might actually be misleading in guiding theories of syntax. As a result, the emphasis in generative grammar has turned from formalisms with restricted generative capacity to those that support more natural expression of the observed regularities of languages. While a variety of distinct approaches have developed, most of them can be characterized as constraint based - the formalism (or formal framework) provides a class of structures and a means of precisely stating constraints on their form, the linguistic theory is then expressed as a system of constraints (or principles) that characterize the class of well-formed analyses of the strings in the language. ${ }^{1}$

As the study of the formal properties of classes of structures defined in such a way falls within domain of Model Theory, it's not surprising that treatments of the meaning of these systems of constraints are typically couched in terms of formal logic (Kasper and Rounds, 1986; Moshier and Rounds, 1987; Kasper and Rounds, 1990; Gazdar et al., 1988; Johnson, 1988; Smolka, 1989; Dawar and Vijay-Shanker, 1990; Carpenter, 1992; Keller, 1993; Rogers and Vijay-Shanker, 1994).

While this provides a model-theoretic interpretation of the systems of constraints produced by these formalisms, those systems are typically built by derivational processes that employ extra-logical mechanisms to combine constraints. More recently, it has become clear that in many cases these mechanisms can be replaced with ordinary logical operations. (See, for instance:

\footnotetext{
${ }^{1}$ This notion of constraint-based includes not only the obvious formalisms, but the formal framework of GB as well.
} 
Johnson (1989), Stabler, Jr. (1992), Cornell (1992), Blackburn, Gardent, and Meyer-Viol (1993), Blackburn and Meyer-Viol (1994), Keller (1993), Rogers (1994), Kracht (1995), and, anticipating all of these, Johnson and Postal (1980).) This approach abandons the notions of grammar mechanism and derivation in favor of defining languages as classes of more or less ordinary mathematical structures axiomatized by sets of more or less ordinary logical formulae. A grammatical theory expressed within such a framework is just the set of logical consequences of those axioms. This step completes the detachment of generative grammar from its procedural roots. Grammars, in this approach, are purely declarative definitions of a class of structures, completely independent of mechanisms to generate or check them. While it is unlikely that every theory of syntax with an explicit derivational component can be captured in this way, ${ }^{2}$ for those that can the logical re-interpretation frequently offers a simplified statement of the theory and clarifies its consequences.

But the accompanying loss of language-theoretic complexity results is unfortunate. While such results may not be useful in guiding syntactic theory, they are not irrelevant. The nature of language-theoretic complexity hierarchies is to classify languages on the basis of their structural properties. The languages in a class, for instance, will typically exhibit certain closure properties (e.g., pumping lemmas) and the classes themselves admit normal forms (e.g., representation theorems). While the linguistic significance of individual results of this sort is open to debate, they at least loosely parallel typical linguistic concerns: closure properties state regularities that are exhibited by the languages in a class, normal forms express generalizations about their structure. So while these may not be the right results, they are not entirely the wrong kind of results. Moreover, since these classifications are based on structural properties and the structural properties of natural language can be studied more or less directly, there is a reasonable expectation of finding empirical evidence falsifying a hypothesis about languagetheoretic complexity of natural languages if such evidence exists.

Finally, the fact that these complexity classes have automata-theoretic characterizations means that results concerning the complexity of natural languages will have implications for the nature of the human language faculty. These automata-theoretic characterizations determine, along one axis, the types of resources required to generate or recognize the lan-

\footnotetext{
${ }^{2}$ Whether there are theories that cannot be captured, at least without explicitly encoding the derivations, is an open question of considerable theoretical interest, as is the question of what empirical consequences such an essential dynamic character might have.
}

guages in a class. The regular languages, for instance, can be characterized by finite-state (string) automata - these languages can be processed using a fixed amount of memory. The context-sensitive languages, on the other had, can be characterized by linear-bounded automata - they can be processed using an amount of memory proportional to the length of the input. The context-free languages are probably best characterized by finite-state tree automata - these correspond to recognition by a collection of processes, each with a fixed amount of memory, where the number of processes is linear in the length of the input and all communication between processes is completed at the time they are spawned. As a result, while these results do not necessarily offer abstract models of the human language faculty (since the complexity results do not claim to characterize the human languages, just to classify them), they do offer lower bounds on certain abstract properties of that faculty. In this way, generative grammar in concert with formal language theory offers insight into a deep aspect of human cognition-syntactic processing-on the basis of observable behavior - the structural properties of human languages.

In this paper we discuss an approach to defining theories of syntax based on $L_{K, P}^{2}$ (Rogers, 1994), a monadic second-order language that has well-defined generative capacity: sets of finite trees are definable within $L_{K, P}^{2}$ iff they are strongly context-free in a particular sense. While originally introduced as a means of establishing language-theoretic complexity results for constraint-based theories, this language has much to recommend it as a general framework for theories of syntax in its own right. Being a monadic second-order language it can capture the (pure) modal languages of much of the existing model-theoretic syntax literature directly; having a signature based on the traditional linguistic relations of domination, immediate domination, linear precedence, etc. it can express most linguistic principles transparently; and having a clear characterization in terms of generative capacity, it serves to re-establish the close connection between generative grammar and formal language theory that was lost in the move away from phrase-structure grammars. Thus, with this framework we get both the advantages of the model-theoretic approach with respect to naturalness and clarity in expressing linguistic principles and the advantages of the grammarbased approach with respect to language-theoretic complexity results.

We look, in particular, at the definitions of a single aspect of each of GPSG and GB. The first of these, Feature Specification Defaults in GPSG, are widely assumed to have an inherently dynamic character. In addition to being purely declarative, our reformalization is considerably simplified wrt the definition 
in Gazdar et al. (1985) ${ }^{3}$ and does not share its misleading dynamic flavor. ${ }^{4}$ We offer this as an example of how re-interpretations of this sort can inform the original theory. In the second example we sketch a definition of chains in GB. This, again, captures a presumably dynamic aspect of the original theory in a static way. Here, though, the main significance of the definition is that it forms a component of a fullscale treatment of a GB theory of English S- and D-Structure within $L_{K, P}^{2}$. This full definition establishes that the theory we capture licenses a strongly context-free language. More importantly, by examining the limitations of this definition of chains, and in particular the way it fails for examples of noncontext-free constructions, we develop a characterization of the context-free languages that is quite natural in the realm of GB. This suggests that the apparent mismatch between formal language theory and natural languages may well have more to do with the unnaturalness of the traditional diagnostics than a lack of relevance of the underlying structural properties.

Finally, while GB and GPSG are fundamentally distinct, even antagonistic, approaches to syntax, their translation into the model-theoretic terms of $L_{K, P}^{2}$ allows us to explore the similarities between the theories they express as well as to delineate actual distinctions between them. We look briefly at two of these issues.

Together these examples are chosen to illustrate the main strengths of the model-theoretic approach, at least as embodied in $L_{K, P}^{2}$, as a framework for studying theories of syntax: a focus on structural properties themselves, rather than on mechanisms for specifying them or for generating or checking structures that exhibit them, and a language that is expressive enough to state most linguistically significant properties in a natural way, but which is restricted enough to have well-defined strong generative capacity.

\section{$2 \quad L_{K, P}^{2}$-The Monadic Second-Order Language of Trees}

$L_{K, P}^{2}$ is the monadic second-order language over the signature including a set of individual constants $(K)$, a set of monadic predicates $(P)$, and binary predicates for immediate domination $(\triangleleft)$, domination $\left(\triangleleft^{*}\right)$, linear precedence $(\prec)$ and equality $(\approx)$. The predicates in $P$ can be understood both as picking out particular subsets of the tree and as (non-exclusive) labels or features decorating the tree. Models for the language are labeled tree do-

\footnotetext{
${ }^{3}$ We will refer to Gazdar et al. (1985) as GKP\&S

${ }^{4}$ We should note that the definition of FSDs in GKP\&S is, in fact, declarative although this is obscured by the fact that it is couched in terms of an algorithm for checking models.
}

mains (Gorn, 1967) with the natural interpretation of the binary predicates. In Rogers (1994) we have shown that this language is equivalent in descriptive power to $S \omega S$ - the monadic second-order theory of the complete infinitely branching tree-in the sense that sets of trees are definable in $\mathrm{S} \omega \mathrm{S}$ iff they are definable in $L_{K, P}^{2}$. This places it within a hierarchy of results relating language-theoretic complexity classes to the descriptive complexity of their models: the sets of strings definable in S1S are exactly the regular sets (Büchi, 1960), the sets of finite trees definable in $\mathrm{S} n \mathrm{~S}$, for finite $n$, are the recognizable sets (roughly the sets of derivation trees of CFGs) (Doner, 1970), and, it can be shown, the sets of finite trees definable in $\mathrm{S} \omega \mathrm{S}$ are those generated by generalized CFGs in which regular expressions may occur on the rhs of rewrite rules (Rogers, 1996b)..$^{5}$ Consequently, languages are definable in $L_{K, P}^{2}$ iff they are strongly context-free in the mildly generalized sense of GPSG grammars.

In restricting ourselves to the language of $L_{K, P}^{2}$ we are restricting ourselves to reasoning in terms of just the predicates of its signature. We can expand this by defining new predicates, even higher-order predicates that express, for instance, properties of or relations between sets, and in doing so we can use monadic predicates and individual constants freely since we can interpret these as existentially bound variables. But the fundamental restriction of $L_{K, P}^{2}$ is that all predicates other than monadic first-order predicates must be explicitly defined, that is, their definitions must resolve, via syntactic substitution, into formulae involving only the signature of $L_{K, P}^{2}$.

\section{Feature Specification Defaults in GPSG}

We now turn to our first application-the definition of Feature Specification Defaults (FSDs) in GPSG. ${ }^{6}$ Since GPSG is presumed to license (roughly) context-free languages, we are not concerned here with establishing language-theoretic complexity but rather with clarifying the linguistic theory expressed by GPSG. FSDs specify conditions on feature values that must hold at a node in a licensed tree unless they are overridden by some other component of the grammar; in particular, unless they are incompatible with either a feature specified by the ID rule licensing the node (inherited features) or a feature required by one of the agreement principles - the Foot Feature Principle (FFP), Head Feature Convention (HFC), or Control Agreement Principle (CAP). It is the fact that the default holds

\footnotetext{
${ }^{5}$ There is reason to believe that this hierarchy can be extended to encompass, at least, a variety of mildly context-sensitive languages as well.

${ }^{6}$ A more complete treatment of GPSG in $L_{K, P}^{2}$ can be found in Rogers (1996c).
} 
just in case it is incompatible with these other components that gives FSDs their dynamic flavor. Note, though, in contrast to typical applications of default logics, a GPSG grammar is not an evolving theory. The exceptions to the defaults are fully determined when the grammar is written. If we ignore for the moment the effect of the agreement principles, the defaults are roughly the converse of the ID rules: a non-default feature occurs iff it is licensed by an ID rule.

It is easy to capture ID rules in $L_{K, P}^{2}$. For instance the rule:

$$
\mathrm{VP} \longrightarrow \mathrm{H}[5], \mathrm{NP}, \mathrm{NP}
$$

can be expressed:

$$
\begin{aligned}
& \mathrm{ID}_{5}\left(x, y_{1}, y_{2}, y_{3}\right) \equiv \\
& \quad \text { Children }\left(x, y_{1}, y_{2}, y_{3}\right) \wedge \mathrm{VP}(x) \wedge \\
& \quad \mathrm{H}\left(y_{1}\right) \wedge\langle\operatorname{SUBCAT}, 5\rangle\left(y_{1}\right) \wedge \mathrm{NP}\left(y_{2}\right) \wedge \mathrm{NP}\left(y_{3}\right),
\end{aligned}
$$

where Children $\left(x, y_{1}, y_{2}, y_{3}\right)$ holds iff the set of nodes that are children of $x$ are just the $y_{i}$ and VP, $\langle\mathrm{SUBCAT}, 5\rangle$, etc. are all members of $P .^{7} \mathrm{~A}$ sequence of nodes will satisfy $\mathrm{ID}_{5}$ iff they form a local tree that, in the terminology of GKP\&S, is induced by the corresponding ID rule. Using such encodings we can define a predicate Free $_{f}(x)$ which is true at a node $x$ iff the feature $f$ is compatible with the inherited features of $x$.

The agreement principles require pairs of nodes occurring in certain configurations in local trees to agree on certain classes of features. Thus these principles do not introduce features into the trees, but rather propagate features from one node to another, possibly in many steps. Consequently, these principles cannot override FSDs by themselves; rather every violation of a default must be licensed by an inherited feature somewhere in the tree. In order to account for this propagation of features, the definition of FSDs in GKP\&S is based on identifying pairs of nodes that co-vary wrt the relevant features in all possible extensions of the given tree. As a result, although the treatment in GKP\&S is actually declarative, this fact is far from obvious.

Again, it is not difficult to define the configurations of local trees in which nodes are required to agree by FFP, CAP, or HFC in $L_{K, P}^{2}$. Let the predicate Propagate $_{f}(x, y)$ hold for a pair of nodes $x$ and $y$ iff they are required to agree on $f$ by one of these principles (and are, thus, in the same local tree). Note that Propagate is symmetric. Following the terminology of GKP\&S, we can identify the set of nodes that are prohibited from taking feature $f$ by the combination of the ID rules, FFP, CAP, and $\mathrm{HFC}$ as the set of nodes that are privileged wrt $f$. This includes all nodes that are not Free for $f$ as well

\footnotetext{
${ }^{7}$ We will not elaborate here on the encoding of categories in $L_{K, P}^{2}$, nor on non-finite ID schema like the iterating co-ordination schema. These present no significant problems.
}

as any node connected to such a node by a sequence of Propagate, links. We, in essence, define this inductively. $\mathrm{P}_{f}^{\prime}(X)$ is true of a set iff it includes all nodes not Free for $f$ and is closed wrt Propagate $_{f}$. $\operatorname{PrivSet}_{f}(X)$ is true of the smallest such set.

$$
\begin{aligned}
& \mathrm{P}_{f}^{\prime}(X) \equiv \\
& \quad(\forall x)\left[\neg \text {Free }_{f}(x) \rightarrow X(x)\right] \wedge \\
& \quad \forall x)\left[(\exists y)\left[X(y) \wedge \text { Propagate }_{f}(x, y)\right] \rightarrow X(x)\right] \\
& \text { PrivSet }_{f}(X) \equiv \\
& \quad \mathrm{P}_{f}^{\prime}(X) \wedge \\
& (\forall Y)\left[\mathrm{P}_{f}^{\prime}(Y) \rightarrow \operatorname{Subset}(X, Y)\right] .
\end{aligned}
$$

There are two things to note about this definition. First, in any tree there is a unique set satisfying PrivSet $_{f}(X)$ and this contains exactly those nodes not Free for $f$ or connected to such a node by Propagate $_{f}$. Second, while this is a first-order inductive property, the definition is a second-order explicit definition. In fact, the second-order quantification of $L_{K, P}^{2}$ allows us to capture any monadic first-order inductively or implicitly definable property explicitly.

Armed with this definition, we can identify individuals that are privileged wrt $f$ simply as the members of PrivSet ${ }_{f}{ }^{8}$

$$
\text { Privileged }_{f}(x) \equiv(\exists X)\left[\text { PrivSet }_{f}(X) \wedge X(x)\right] .
$$

One can define Privileged $\neg f(x)$ which holds whenever $x$ is required to take the feature $f$ along similar lines.

These, then, let us capture FSDs. For the default [-INV], for instance, we get:

$$
(\forall x)\left[\neg \operatorname{Privileged}_{[-\mathrm{INV}]}(x) \rightarrow[-\operatorname{INV}](x)\right] .
$$

For [BAR 0] J [PAS] (which says that [Bar 0] nodes are, by default, not marked passive), we get:

$$
\begin{aligned}
& (\forall x)\left[\left([\operatorname{BAR} 0](x) \wedge \neg \text { Privileged }_{\neg[\mathrm{PAS}]}(x)\right) \rightarrow\right. \\
& \quad \neg[\mathrm{PAS}](x)] .
\end{aligned}
$$

The key thing to note about this treatment of FSDs is its simplicity relative to the treatment of GKP\&S. The second-order quantification allows us to reason directly in terms of the sequence of nodes extending from the privileged node to the local tree that actually licenses the privilege. The immediate benefit is the fact that it is clear that the property of satisfying a set of FSDs is a static property of labeled trees and does not depend on the particular strategy employed in checking the tree for compliance.

\footnotetext{
${ }^{8}$ We could, of course, skip the definition of PrivSet $f$ and define Privileged $f(x)$ as $(\forall X)\left[\mathrm{P}^{\prime}(X) \rightarrow X(x)\right]$, but we prefer to emphasize the inductive nature of the definition.
} 


\section{Chains in GB}

The key issue in capturing GB theories within $L_{K, P}^{2}$ is the fact that the mechanism of free-indexation is provably non-definable. Thus definitions of principles that necessarily employ free-indexation have no direct interpretation in $L_{K, P}^{2}$ (hardly surprising, as we expect GB to be capable of expressing noncontext-free languages). In many cases, though, references to indices can be eliminated in favor of the underlying structural relationships they express. ${ }^{9}$ The most prominent example is the definition of the chains formed by move- $\alpha$. The fundamental problem here is identifying each trace with its antecedent without referencing their index. Accounts of the licensing of traces that, in many cases of movement, replace co-indexation with government relations have been offered by both Rizzi (1990) and Manzini (1992). The key element of these accounts, from our point of view, is that the antecedent of a trace must be the closest antecedent-governor of the appropriate type. These relationships are easy to capture in $L_{K, P}^{2}$. For $\overline{\mathrm{A}}$-movement, for instance, we have:

$$
\begin{aligned}
& \overline{\mathrm{A}} \text {-Antecedent-Governs }(x, y) \equiv \\
& \neg \mathrm{A}-\operatorname{pos}(x) \wedge \mathrm{C}-\operatorname{Commands}(x, y) \wedge \mathrm{F} \cdot \mathrm{Eq}(x, y) \wedge \\
& -x \text { is a potential antecedent in an } \\
& \overline{\mathrm{A}} \text {-position } \\
& \neg(\exists z)[\text { Intervening-Barrier }(z, x, y)] \wedge \\
& - \text { no barrier intervenes } \\
& \neg(\exists z)[\operatorname{Spec}(z) \wedge \neg \mathrm{A}-\operatorname{pos}(z) \wedge \\
& \quad \mathrm{C}-\operatorname{Commands}(z, x) \wedge \operatorname{Intervenes}(z, x, y)] \\
& - \text { minimality is respected }
\end{aligned}
$$

where $\mathrm{F} . \mathrm{Eq}(x, y)$ is a conjunction of biconditionals that assures that $x$ and $y$ agree on the appropriate features and the other predicates are are standard GB notions that are definable in $L_{K, P}^{2}$.

Antecedent-government, in Rizzi's and Manzini's accounts, is the key relationship between adjacent members of chains which are identified by nonreferential indices, but plays no role in the definition of chains which are assigned a referential index. ${ }^{10}$ Manzini argues, however, that referential chains cannot overlap, and thus we will never need to distinguish multiple referential chains in any single context. Since we can interpret any bounded number of indices simply as distinct labels, there is no difficulty in identifying the members of referential chains in $L_{K, P}^{2}$. On these and similar grounds we can extend these accounts to identify adjacent members of referential chains, and, at least in the case of English,

\footnotetext{
${ }^{9}$ More detailed expositions of the interpretation of GB in $L_{K, P}^{2}$ can be found in Rogers (1996a), Rogers (1995), and Rogers (1994).

${ }^{10}$ This accounts for subject/object asymmetries.
}

of chains of head movement and of rightward movement. This gives us five mutually exclusive relations which we can combine into a single link relation that must hold between every trace and its antecedent:

$$
\begin{aligned}
& \operatorname{Link}(x, y) \equiv \operatorname{A}-\operatorname{Link}(x, y) \vee \overline{\mathrm{A}}-\overline{\operatorname{Ref}}-\operatorname{Link}(x, y) \vee \\
& \overline{\mathrm{A}}-\operatorname{Ref}-\operatorname{Link}(x, y) \vee \mathrm{X}^{0}-\operatorname{Link}(x, y) \vee
\end{aligned}
$$

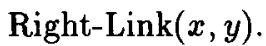

The idea now is to define chains as sequences of nodes that are linearly ordered by Link, but before we can do this there is still one issue to resolve. While minimality ensures that every trace must have a unique antecedent, we may yet admit a single antecedent that licenses multiple traces. To rule out this possibility, we require chains to be closed wrt the link relation, i.e., every chain must include every node that is related by Link to any node already in the chain. Our definition, then, is in essence the definition, in GB terms, of a discrete linear order with endpoints, augmented with this closure property.

$$
\begin{aligned}
& \text { Chain }(X) \equiv \\
& (\exists ! x)[X(x) \wedge \text { Target }(x)] \wedge \\
& -X \text { contains exactly one Target } \\
& (\exists ! x)[X(x) \wedge \operatorname{Base}(x)] \wedge \\
& - \text { and one Base } \\
& (\forall x)[X(x) \wedge \neg \text { Target }(x) \rightarrow \\
& (\exists ! y)[X(y) \wedge \operatorname{Link}(y, x)]] \wedge \\
& \text { - All non-Target have a unique an- } \\
& \text { tecedent in } X \\
& (\forall x)[X(x) \wedge \neg \operatorname{Base}(x) \rightarrow \\
& (\exists ! y)[X(y) \wedge \operatorname{Link}(x, y)]] \wedge \\
& \text {-All non-Base have a unique suc- } \\
& \text { cessor in } X \\
& (\forall x, y)[X(x) \wedge(\text { Link }(x, y) \vee \operatorname{Link}(y, x)) \rightarrow \\
& X(y)] \\
& -X \text { is closed wrt the Link relation }
\end{aligned}
$$

Note that every node will be a member of exactly one (possibly trivial) chain.

The requirement that chains be closed wrt Link means that chains cannot overlap unless they are of distinct types. This definition works for English because it is possible, in English, to resolve chains into boundedly many types in such a way that no two chains of the same type ever overlap. In fact, it fails only in cases, like head-raising in Dutch, where there are potentially unboundedly many chains that may overlap a single point in the tree. Thus, this gives us a property separating GB theories of movement that license strongly context-free languages from those that potentially don't-if we can establish a fixed bound on the number of chains that can overlap, then the definition we sketch here will suffice to capture the theory in $L_{K, P}^{2}$ and, consequently, the theory licenses only strongly context-free languages. 
This is a reasonably natural diagnostic for contextfreeness in GB and is close to common intuitions of what is difficult about head-raising constructions; it gives those intuitions theoretical substance and provides a reasonably clear strategy for establishing context-freeness.

\section{A Comparison and a Contrast}

Having interpretations both of GPSG and of a $G B$ account of English in $L_{K, P}^{2}$ provides a certain amount of insight into the distinctions between these approaches. For example, while the explanations of filler-gap relationships in GB and GPSG are quite dramatically dissimilar, when one focuses on the structures these accounts license one finds some surprising parallels. In the light of our interpretation of antecedent-government, one can understand the role of minimality in Rizzi's and Manzini's accounts as eliminating ambiguity from the sequence of relations connecting the gap with its filler. In GPSG this connection is made by the sequence of agreement relationships dictated by the Foot Feature Principle. So while both theories accomplish agreement between filler and gap through marking a sequence of elements falling between them, the GB account marks as few as possible while the GPSG account marks every node of the spine of the tree spanning them. In both cases, the complexity of the set of licensed structures can be limited to be strongly context-free iff the number of relationships that must be distinguished in a given context can be bounded.

One finds a strong contrast, on the other hand, in the way in which GB and GPSG encode language universals. In GB it is presumed that all principles are universal with the theory being specialized to specific languages by a small set of finitely varying parameters. These principles are simply properties of trees. In terms of models, one can understand $G B$ to define a universal language-the set of all analyses that can occur in human languages. The principles then distinguish particular sub-languages-the head-final or the pro-drop languages, for instance. Each realized human language is just the intersection of the languages selected by the settings of its parameters. In GPSG, in contrast, many universals are, in essence, closure properties that must be exhibited by human languages-if the language includes trees in which a particular configuration occurs then it includes variants of those trees in which certain related configurations occur. Both the ECPO principle and the metarules can be understood in this way. Thus while universals in GB are properties of trees, in GPSG they tend to be properties of sets of trees. This makes a significant difference in capturing these theories model-theoretically; in the GB case one is defining sets of models, in the GPSG case one is defining sets of sets of models. It is not at all clear what the linguistic significance of this distinction is; one particularly interesting question is whether it has empirical consequences. It is only from the model-theoretic perspective that the question even arises.

\section{Conclusion}

We have illustrated a general formal framework for expressing theories of syntax based on axiomatizing classes of models in $L_{K, P}^{2}$. This approach has a number of strengths. First, as should be clear from our brief explorations of aspects of GPSG and GB, re-formalizations of existing theories within $L_{K, P}^{2}$ can offer a clarifying perspective on those theories, and, in particular, on the consequences of individual components of those theories. Secondly, the framework is purely declarative and focuses on those aspects of language that are more or less directly observable--their structural properties. It allows us to reason about the consequences of a theory without hypothesizing a specific mechanism implementing it. The abstract properties of the mechanisms that might implement those theories, however, are not beyond our reach. The key virtue of descriptive complexity results like the characterizations of language-theoretic complexity classes discussed here and the more typical characterizations of computational complexity classes (Gurevich, 1988; Immerman, 1989) is that they allow us to determine the complexity of checking properties independently of how that checking is implemented. Thus we can use such descriptive complexity results to draw conclusions about those abstract properties of such mechanisms that are actually inferable from their observable behavior. Finally, by providing a uniform representation for a variety of linguistic theories, it offers a framework for comparing their consequences. Ultimately it has the potential to reduce distinctions between the mechanisms underlying those theories to distinctions between the properties of the sets of structures they license. In this way one might hope to illuminate the empirical consequences of these distinctions, should any, in fact, exist.

\section{References}

Blackburn, Patrick, Claire Gardent, and Wilfried Meyer-Viol. 1993. Talking about trees. In $E A C L$ 93, pages 21-29. European Association for Computational Linguistics.

Blackburn, Patrick and Wilfried Meyer-Viol. 1994. Linguistics, logic, and finite trees. Bulletin of the IGPL, 2(1):3-29, March.

Büchi, J. R. 1960. Weak second-order arithmetic and finite automata. Zeitschrift für mathematische Logik und Grundlagen der Mathematik, 6:6692 . 
Carpenter, Bob. 1992. The Logic of Typed Feature Structures; with Applications to Unification Grammars, Logic Programs and Constraint Resolution. Number 32 in Cambridge Tracts in Theoretical Computer Science. Cambridge University Press.

Cornell, Thomas Longacre. 1992. Description Theory, Licensing Theory, and Principle-Based Grammars and Parsers. Ph.D. thesis, University of California Los Angeles.

Dawar, Anuj and K. Vijay-Shanker. 1990. An interpretation of negation in feature structure descriptions. Computational Linguistics, 16(1):11-21.

Doner, John. 1970. Tree acceptors and some of their applications. Journal of Computer and System Sciences, 4:406-451.

Gazdar, Gerald, Ewan Klein, Geoffrey Pullum, and Ivan Sag. 1985. Generalized Phrase Structure Grammar. Harvard University Press.

Gazdar, Gerald, Geoffrey Pullum, Robert Carpenter, Ewan Klein, T. E. Hukari, and R. D. Levine. 1988. Category structures. Computational Linguistics, 14:1-19.

Gorn, Saul. 1967. Explicit definitions and linguistic dominoes. In John F. Hart and Satoru Takasu, editors, Systems and Computer Science, Proceedings of the Conference held at Univ. of Western Ontario, 1965. Univ. of Toronto Press.

Gurevich, Yuri. 1988. Logic and the challenge of computer science. In E. Börger, editor, Current Trends in Theoretical Computer Science. Computer Science Press, chapter 1, pages 1-57.

Immerman, Neil. 1989. Descriptive and computational complexity. In Proceedings of Symposia in Applied Mathematics, pages 75-91. American Mathematical Society.

Johnson, David E. and Paul M. Postal. 1980. Arc Pair Grammar. Princeton University Press, Princeton, New Jersey.

Johnson, Mark. 1988. Attribute-Value Logic and the Theory of Grammar. Number 16 in CSLI Lecture Notes. Center for the Study of Language and Information, Stanford, CA.

Johnson, Mark. 1989. The use of knowledge of language. Journal of Psycholinguistic Research, 18(1):105-128.

Kasper, Robert T. and William C. Rounds. 1986. A logical semantics for feature structures. In Proceedings of the 24th Annual Meeting of the Association for Computational Linguistics.

Kasper, Robert T. and William C. Rounds. 1990. The logic of unification in grammar. Linguistics and Philosophy, 13:35-58.
Keller, Bill. 1993. Feature Logics, Infinitary Descriptions and Grammar. Number 44 in CSLI Lecture Notes. Center for the Study of Language and Information.

Kracht, Marcus. 1995. Syntactic codes and grammar refinement. Journal of Logic, Language, and Information, 4:41-60.

Manzini, Maria Rita. 1992. Locality: A Theory and Some of Its Empirical Consequences. MIT Press, Cambridge, Ma.

Moshier, M. Drew and William C. Rounds. 1987. A logic for partially specified data structures. In ACM Symposium on the Principles of Programming Languages.

Rizzi, Luigi. 1990. Relativized Minimality. MIT Press.

Rogers, James. 1994. Studies in the Logic of Trees with Applications to Grammar Formalisms. Ph.D. dissertation, Univ. of Delaware.

Rogers, James. 1995. On descriptive complexity, language complexity, and GB. In Patrick Blackburn and Maarten de Rijke, editors, Specifying Syntactic Structures. In Press. Also available as IRCS Technical Report 95-14. cmp-lg/9505041.

Rogers, James. 1996a. A Descriptive Approach to Language-Theoretic Complexity. Studies in Logic, Language, and Information. CSLI Publications. To appear.

Rogers, James. 1996b. The descriptive complexity of local, recognizable, and generalized recognizable sets. Technical report, IRCS, Univ. of Pennsylvania. In Preparation.

Rogers, James. 1996c. Grammarless phrasestructure grammar. Under Review.

Rogers, James and K. Vijay-Shanker. 1994. Obtaining trees from their descriptions: An application to tree-adjoining grammars. Computational Intelligence, 10:401-421.

Smolka, Gert. 1989. A feature logic with subsorts. LILOG Report 33, IBM Germany, Stuttgart.

Stabler, Jr., Edward P. 1992. The Logical Approach to Syntax. Bradford. 